EPJ Web of Conferences 16, 07006 (2011)

DOI: $10.1051 /$ epjconf/20111607006

(C) Owned by the authors, published by EDP Sciences, 2011

\title{
CHIRON - A new high resolution spectrometer for CTIO
}

\author{
C. Schwab ${ }^{1}$, J.F.P. Spronck ${ }^{2}$, A. Tokovinin ${ }^{3}$, D.A. Fischer ${ }^{2}$ and G.W. Marcy ${ }^{4}$ \\ ${ }^{1}$ ZAH-Landessternwarte, Heidelberg, Germany \\ ${ }^{2}$ Yale University, New Haven, CT, USA \\ ${ }^{3}$ CTIO, La Serena, Chile \\ ${ }^{4}$ University of California, Berkeley, CA, USA
}

\begin{abstract}
Small telescopes can play an important role in the search for exoplanets because they offer an opportunity for high cadence observations that are not possible with large aperture telescopes. However, there is a shortage of high resolution spectrometers for precision Doppler planet searches. We report on an innovative design for CHIRON, an inexpensive spectrometer that we are building for the $1.5-\mathrm{m}$ telescope at CTIO in Chile.

The resolution will be $\mathrm{R}>80.000$, the spectral format spanning 410 to $880 \mathrm{~nm}$. The total throughput of the telescope and spectrometer will be better than $12 \%$, comparable with the efficiency of state-of-the-art spectrometers. The design is driven by the requirements for precision Doppler searches for exoplanets using an iodine cell.

The optical layout is a classical echelle with $140 \mathrm{~mm}$ beam size. The bench-mounted spectrometer will be fibre-fed followed by an image slicer. An apochromatic refractor is used as the camera. Image quality and throughput of the design are excellent over the full spectral range. Extensive use of commercially available components and avoidance of complicated custom optics are key for quick and resource-efficient implementation.
\end{abstract}

\section{OPTICAL DESIGN}

Our optical layout is a pseudo-Littrow configuration. The spectrograph optics fit comfortably on a standard $1 \mathrm{~m} \times 1.5 \mathrm{~m}$ optical table (Fig. 1). The CCD is mounted separately to avoid transmitting variable mechanical and thermal loads to the main structure. We plan to use commercially available kinematic mounts. For the collimator we foresee an on-axis parabolic mirror, facilitating ease of alignment, and giving high optical quality. The necessary central obstruction is minimal.

The fibre feed already in place at the telescope uses a $100 \mu \mathrm{m}$ fibre at $\mathrm{f} / 5$. Slicing the fibre image three times gives a projected "slit" of $50 \times 450 \mu \mathrm{m}$, well matched to the CCD pixel size and our envisaged resolution. We adopted the Bowen-Walraven design for the slicer. Mirrors with an enhanced metal coating are used rather than the total internal reflection in prisms commonly used in other slicers. Due to the high reflectivity of modern coatings, the efficiency of such a slicer is very good. It is very flexible as the gap can be adjusted. A prototype showed excellent results (Fig. 2).

The grating is an existing R2 echelle with $31.6 \mathrm{gr} / \mathrm{mm}$. For the camera we use an $140 \mathrm{~mm}$ oil-spaced triplet lens plus a two-element field flattener with a focal length of $1000 \mathrm{~mm}$, originally intended as a high-end amateur telescope, from TEC. Image quality over the whole illuminated circle of $85 \mathrm{~mm}$ diameter is very good. The prevalent aberration is chromatic defocus, which we can correct by tilting the CCD slightly. State-of-the-art anti-reflection coatings come as a standard feature.

On an a $4 \mathrm{k}$ by $4 \mathrm{k}$ CCD with $15 \mu \mathrm{m}$ pixels the spectral format spans from 410 to $880 \mathrm{~nm}$ in 73 orders. Assuming a single cross-disperser prism of LF7 glass, the inter-order separation varies by a factor of 1.9. The resulting resolution is 83.000 , slighly oversampled with 3 pixels per slit width.

This is an Open Access article distributed under the terms of the Creative Commons Attribution-Noncommercial License 3.0, which permits unrestricted use, distribution, and reproduction in any noncommercial medium, provided the original work is properly cited. 


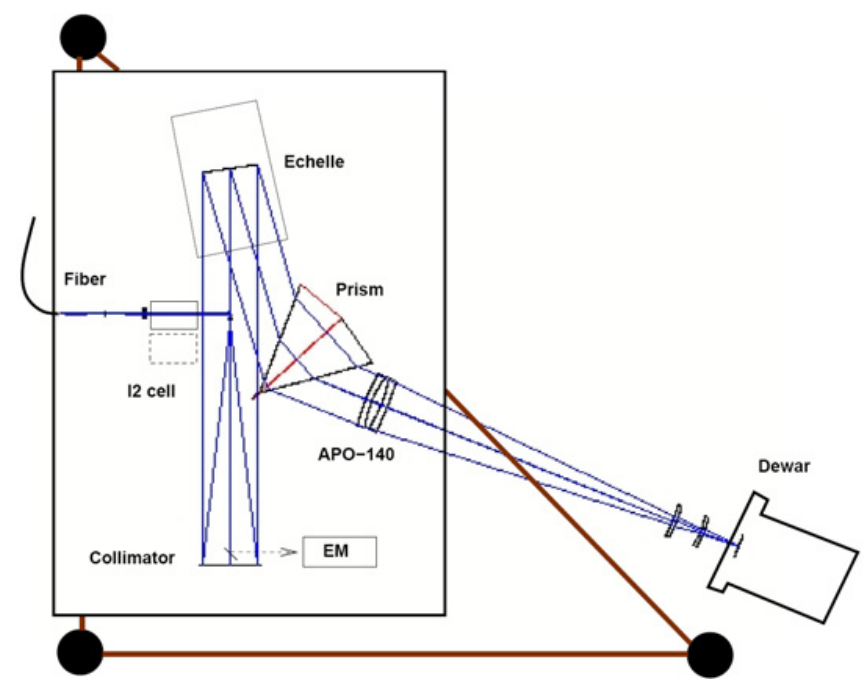

Figure 1. Optical and mechanical layout of the spectrometer. The rectangle outlines a standard optical table of $1 \times 1.5 \mathrm{~m}$. A small mirror in front of the collimator deviates $1 \%$ of the light to the exposure meter.
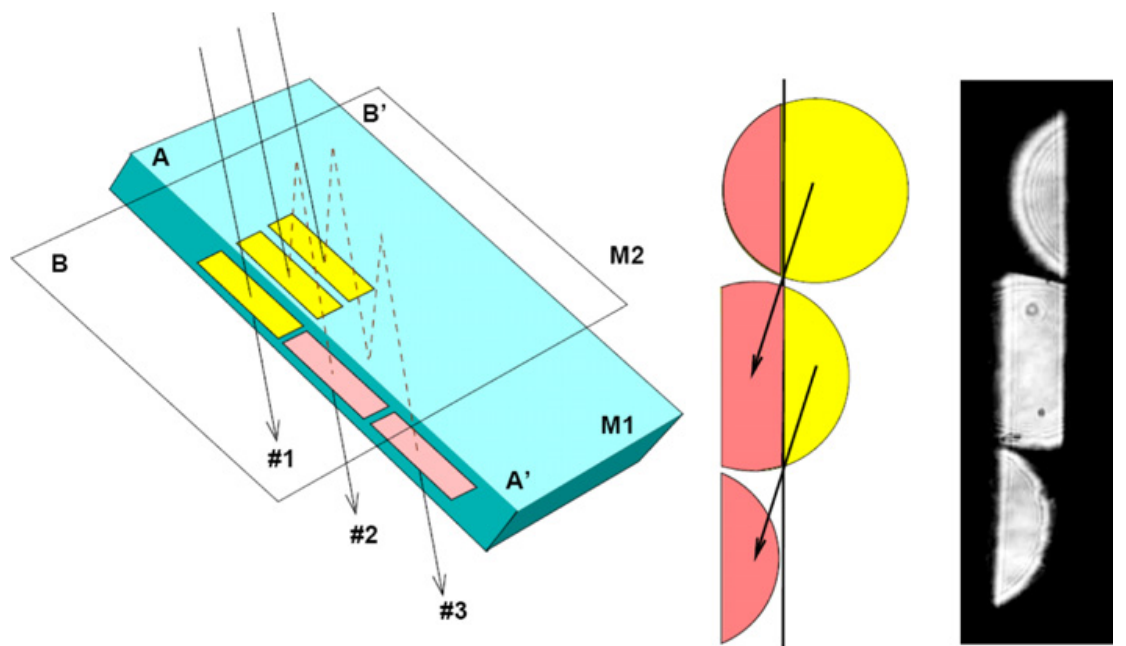

Figure 2. The Bowen-Walraven type image slicer. The resulting slices from a prototype are shown on the right.

We estimate the throughput of the system at the blaze peak, from the atmosphere to the CCD, to be $12 \%$ at $500 \mathrm{~nm}$. This includes the existing echelle $(65 \%)$, the two telescope mirrors (75\%), and the CCD. The fibre link, including relay optics, image slicer and seeing effects, is conservatively assumed to have a transmission of $42 \%$. Further improvement can be achieved with a new grating or recoating of the telescope mirrors.

\section{CONCLUSIONS}

We presented a simple, yet efficient spectrograph design. All our main design goals are met. Throughput and image quality are very good over the whole spectral range. This can be achieved using commercially 
Research, Science and Technology of Brown Dwarfs and Exoplanets

available optics, resulting in a state-of-the-art instrument within a manageable budget. The high throughput allows for very efficient use of observing time; coupled with the high resolution, this will be a very valuable addition to the existing RV planet search facilities.

We want to thank Andrew Rakich for helpful discussions, and our design reviewers Andrew Szentgyorgyi and John Hearnshaw for their constructive comments. Fischer acknowledges funding from the NSF MRI program AST-0923441. 